\title{
MARCADORES SONOGRAFICOS DE CARDIOPATIAS CONGENITAS. INTERRUPCION DE LA VENA CAVA INFERIOR: A PROPOSITO DE NUESTRA EXPERIENCIA Y RESULTADOS ${ }^{*}$
}

\author{
Drs. Fernando Viñals L., Marcela Muñoz F., Arrigo Giuliano B. \\ Centro AGB Ultrasonografía. Clínica Sanatorio Alemán, Concepción
}

\section{RESUMEN}

Consideramos marcadores de cardiopatías congénitas al grupo de lesiones que, pudiendo ser variantes normales, se asocian a defectos cardíacos. En esta primera publicación destacamos la importancia del diagnóstico de la interrupción de la vena cava inferior ( $\mathrm{VCl}$ y y su continuación por el sistema azygos, como un marcador de isomerismo auricular y poliesplenia. En toda nuestra serie de cardiopatías congénitas (Cc), hemos diagnosticado 5 anomalías de la $\mathrm{VCl}, 4$ de ellas correspondieron a interrupciones sin Cc asociadas. Reportamos los hallazgos in utero y el seguimiento postnatal de estos casos, la técnica más apropiada para su pesquisa y su asociación a Cc.

\section{PALABRAS CLAVES: Diagnóstico prenatal, cardiopatías congénitas}

\section{SUMMARY}

We consider markers of congenital hear disease a group of anomalies which could be a normal variant in general population but might be associated to structural defects of the fetal heart. One of those marks is the interruption of the inferior vena cava with azygos vein continuation. Among 5 abnormalities of the inferior vena cava diagnosed in our series, we found 4 interruption of the IVC. We discuss in utero abnormalities and follow-up. We propose the technique to screen a group of congenital heart disease with the vascular arrangements in the fetal abdomen.

KEY WORDS: Interruption inferior vena cava, azygos vein, isomerism of the atrial appendages congenital heart disease, prenatal diagnosis

\section{INTRODUCCION}

Definiremos como marcadores sonográficos de Cardiopatías congénitas aquel grupo de lesiones que, pudiendo ser variantes normales en la población general, se asocian a defectos estructurales cardíacos. El hallazgo ecográfico de estos permite seleccionar aquellos fetos que requieren un estudio ecocardiográfico detallado, además de un apropiado consejo. Incluimos en este grupo de lesiones a:

1. Incremento de la transluscencia nucal entre las 11-14 semanas de gestación.

2. Interrupción de la vena cava inferior y continuación por sistema azygos.

*Trabajo recibido en julio de 2002 y aceptado para publicación por el Comité Editor en septiembre de 2002. 
3. Persistencia de la vena cava superior izquierda y desembocadura en seno coronario.

4. Desproporción de las cavidades cardíacas.

Considerando que la aplicación y utilidad del primero de los marcadores ha sido extensamente reportada $(1,2)$, nos referiremos en esta primera publicación a la interrupción de la vena cava inferior.

Las alteraciones de las conexiones del sistema venoso sistémico incluyen las anomalías de la vena cava superior derecha e izquierda, las anomalías del seno coronario y las anomalías de la vena cava inferior $(\mathrm{VCl})$. La alteración más frecuente de la vena cava inferior es su interrupción (3). La VCl se forma entre las 6-8 semanas de gestación, a partir de la vena hepática, vena subcardinal derecha, anastomosis subcardinal y vena supracardinal derecha. La falla en la unión de la vena subcardinal derecha y la vena hepática determinan una deficiencia de la $\mathrm{VCl}$ hacia las venas renales. Por este motivo, la anastomosis entre la vena subcardinal y supracardinal derecha recogen la sangre de la par-

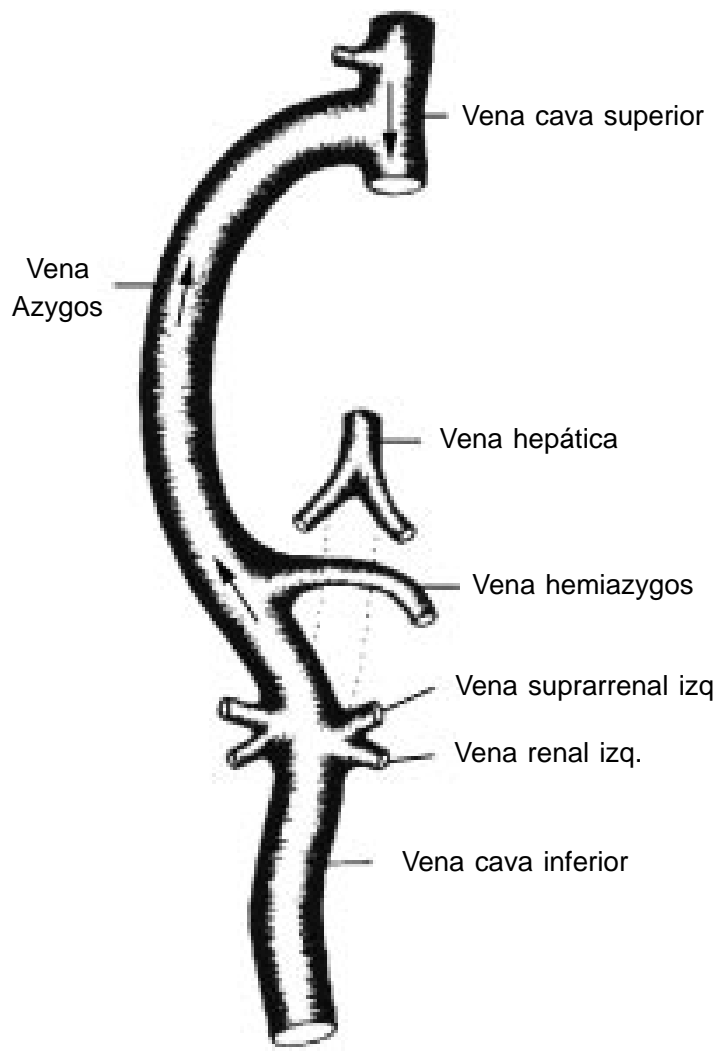

Figura 1. Esquema que demuestra la interrupción de la vena inferior entre las venas renales y las venas hepáticas. La sangre retorna al corazón a través de la vena azygos y vena cava superior. te inferior del cuerpo, derivándola hacia la vena azygos (Figura 1) (4).

La interrupción de la vena cava inferior infrahepática y su continuación por el sistema venoso azygos (Az) se define como la ausencia de la $\mathrm{VCl}$ entre las venas renales y las venas hepáticas. Por sobre las venas renales, el drenaje venoso sistémico retorna por la vena azygos a través de su curso habitual, a derecha de la columna vertebral (5). Posteriormente, la vena azygos ingresa al tórax por el hiato aórtico del diafragma y se une a la vena cava superior justo sobre su desembocadura en la aurícula derecha, en el espacio paratraqueal derecho. Las venas hepáticas desembocan directamente a la aurícula derecha (3).

La interrupción de la $\mathrm{VCl}$ con continuación azygos es la anormalidad más común de este sistema venoso (6). Se asocia frecuentemente a un drenaje directo de las venas hepáticas hacia la aurícula derecha y es habitualmente parte del síndrome de poliesplenia (isomerismo auricular o síndrome de heterotaxia). La interrupción de la vena cava inferior está presente en cerca del $80 \%$ de los casos de isomerismo auricular izquierdo, que se asocia con bloqueo aurículo-ventricular completo e hidrops (7). También a poliesplenia, incluso en ausencia de defectos intracardíacos (8). Por este motivo, es considerado un excelente marcador de estas anomalías (9).

La interrupción es extremadamente rara en pacientes con corazón normal. Se ha reportado en una frecuencia entre $0,6 \%$ hasta $2,9 \%$ en individuos con caridopatías congénitas que han sido estudiados mediante cateterismo cardíaco (10).

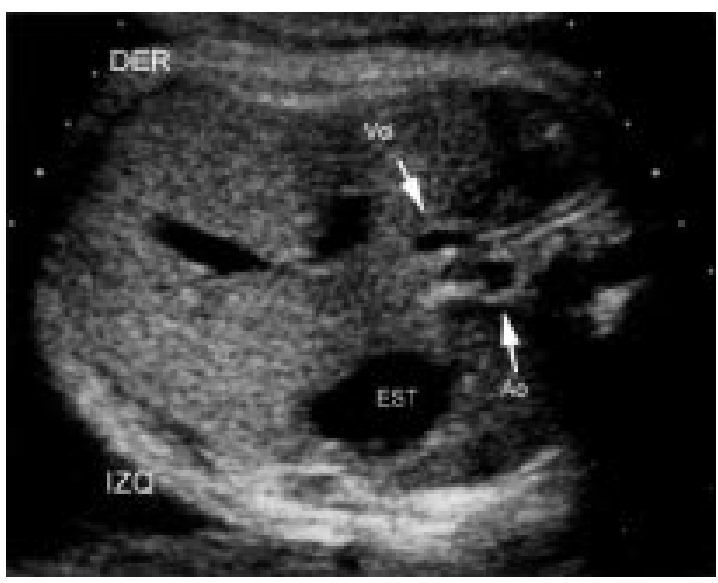

Figura 2. Situs abdominal normal. La vena cava inferior (Vci) se localiza por delante y a derecha de la aorta abdominal (Ao). 
El diagnóstico de esta anomalía es posible in utero. El plano de sección ecográfico más importante para su sospecha diagnóstica es la visión transversa del abdomen fetal, reconocido por todos los operadores por la medición de los diámetros para el cálculo de peso fetal. Sin embargo, a este nivel deben también evaluarse la posición del estómago y la relación entre la aorta abdominal y la vena cava inferior. Estos últimos definen el situs auricular. La localización normal de las estructuras vasculares a este nivel es: la aorta abdominal por delante y a izquierda del cuerpo vertebral; la vena cava inferior por delante de la aorta y a derecha de la línea media imaginaria trazada entre la columna vertebral y la insersión del cordón umbilical (Figura 2). Ambos vasos son similares en su tamaño, destacando la aorta por ser más pulsátil. Al desplazar el transductor hacia cefálica y sin angularlo, la $\mathrm{VCl}$ se continúa con la aurícula derecha. La desembocadura de esta vena se produce en la porción póstero-lateral del septum auricular, cercano a la apertura del foramen oval.

Nuestro interés es reportar los casos de interrupción de $\mathrm{VCl}$ diagnosticados in utero, la técnica más apropiada para su pesquisa y su asociación con cardiopatías congénitas.

\section{MATERIAL Y METODO}

Las pacientes evaluadas en nuestro Centro, predominantemente de bajo riesgo de dar a luz recién nacidos (RN) con cardiopatía congénitas (Cc), son examinadas ecográficamente adicionando un estudio extendido del corazón fetal que incluye una secuencia de secciones transversas desde el abdomen fetal hasta el mediastino superior, en blanco y negro y adicionando Doppler color, según técnica descrita $(11,12)$. En caso de sospecharse una alteración estructural del corazón, se efectuaron inmediatamente las secciones ecocardiográficas necesarias para completar su estudio. Se obtuvo un respaldo digital de todos los casos de Cc diagnosticadas, para su estudio y análisis posterior.

Desde el año 2000 a la fecha, diagnosticamos 5 anomalías de la vena cava inferior. Todos los recién nacidos fueron evaluados por cardiólogo infantil y seguidos hasta los 3 meses de vida a lo menos, efectuándose ecotomografía abdominal y ecocardiografía postnatal como estudios complementarios.

La vena cava inferior fue estudiada mediante secciones transversas y sagitales del abdomen fetal. El situs vascular normal visible en el primero de los planos de sección, a nuestro juicio el más reproducible, tal como fuera detallado en la Figura 2. En caso de existir una interrupción de la vena cava superior y continuación azygos, se deben incluir la visión de 4 cámaras y la visión de 3 vasos del mediastino fetal como complementos para el diagnóstico y documentación apropiada del caso. La descripción de los reparos anatómicos en estos planos de sección ha sido descrita anteriormente (13).

\section{RESULTADOS}

En este período de estudio, diagnosticamos 5 anomalías de la vena cava inferior y continuación azygos, sin defectos estructurales cardíacos pesquisados in utero. El quinto caso correspondió a un feto portador de un isomerismo auricular derecho con una anomalías de posición de la vena cava inferior a nivel del situs abdominal y es incluida en el presente trabajo para consignar la importancia del plano de sección abdominal en la sospecha diagnóstica de anomalías relacionadas con esta estructura venosa.

El detalle de los casos de interrupción de la $\mathrm{VCl}$ y continuación azygos aislada se analizan en las Tablas I y II.

Tabla I

DISTRIBUCION DE CASOS DE INTERRUPCION DE VCI Y CONTINUACION AZYGOS DIAGNOSTICADOS. VISUALIZACION DE VASOS EN 4 CAMARAS, ABDOMEN TRANSVERSO, ABDOMEN SAGITAL Y 3 VASOS (Figura 6)

\begin{tabular}{lccccccc}
\hline Caso & Año & Edad & $\begin{array}{c}E G \\
\text { (sem.) }\end{array}$ & $\begin{array}{c}2 \text { casos } \\
\text { (4 cám.) }\end{array}$ & $\begin{array}{c}\text { Azygos } \\
\text { (transverso) }\end{array}$ & $\begin{array}{c}2 \text { vasos } \\
\text { (sagital) }\end{array}$ & $\begin{array}{c}\text { Desembocadura } \\
\text { (enVCS derecha) }\end{array}$ \\
\hline 1 & 2001 & 30 & 35 & Sí & Sí & Sí & No \\
2 & 2001 & 27 & $30^{*}$ & Sí & Sí & Sí & Sí \\
3 & 2001 & 36 & $3^{*}$ & Sí & Sí & Sí & Sí \\
4 & 2002 & 28 & $28^{*}$ & Sí & Sí & Sí & Sí \\
\hline
\end{tabular}

2 vasos: aorta abdominal y vena azygos; sí: visualizada; no: no visualizada.

*: 1er control ecográfico en nuestro Centro. 
Tabla II

\section{DESCRIPCION DE CASOS DE INTERRUPCION DE VCI. RESULTADOS POSTNATAL Y DE ESTUDIOS ECOGRAFICOS Y ECOCARDIOGRAFICOS}

\begin{tabular}{lccccc}
\hline Caso & Sexo & Anomalías asociadas & $\begin{array}{c}\text { Resultado } \\
\text { (vivo/asintomático) }\end{array}$ & $\begin{array}{c}\text { Ecografía } \\
\text { abdominal }\end{array}$ & $\begin{array}{c}\text { Ecocardiografía } \\
\text { postparto }\end{array}$ \\
\hline 1 & $\mathrm{~F}$ & Monorrea & Vivo/asint. & $-\mathrm{VCl} / \mathrm{azy}+$ & Normal \\
2 & $\mathrm{~F}$ & No & Vivo/asint. & $\mathrm{VCl} / \mathrm{azy}-$ & Normal \\
3 & $\mathrm{M}$ & No & Vivo/asint. & $\mathrm{VCl} / \mathrm{azy}+$ & Normal \\
4 & $\mathrm{~F}$ & Hipertrofia mioc. & Vivo asint. & $\mathrm{VCl} / \mathrm{azy}+$ & No \\
\hline
\end{tabular}

VCI: Porción hepática de VCI no visualizada; azy+: azygos visualizada en ecografía.

En todos los casos, el diagnóstico se sospechó por la ausencia de la $\mathrm{VCl}$ en su porción hepática, por la visualización de un vaso no pulsátil a derecha de la columna y más atrás que la aorta abdominal (Figura 3).

Cuando existe una continuación azygos de la $\mathrm{VCl}$ hacia la vena cava superior derecha, la vena azygos puede visualizarse también por detrás del corazón fetal. Clásicamente, por detrás de la aurícula izquierda se observa siempre la aorta como único vaso evidente visible en 4 cámaras. En casos de continuación azygos, se visualiza además la vena en este plano de sección (Figura 4). En los 4 casos reportados, el diagnóstico de interrupción de la $\mathrm{VCl}$ se sospechó también por la detección de 2 vasos de tamaño similar localizados en la parte posterior del tórax, detrás del corazón.

A nivel del mediastino superior fetal, particularmente en la visión de 3 vasos, se ordenan en

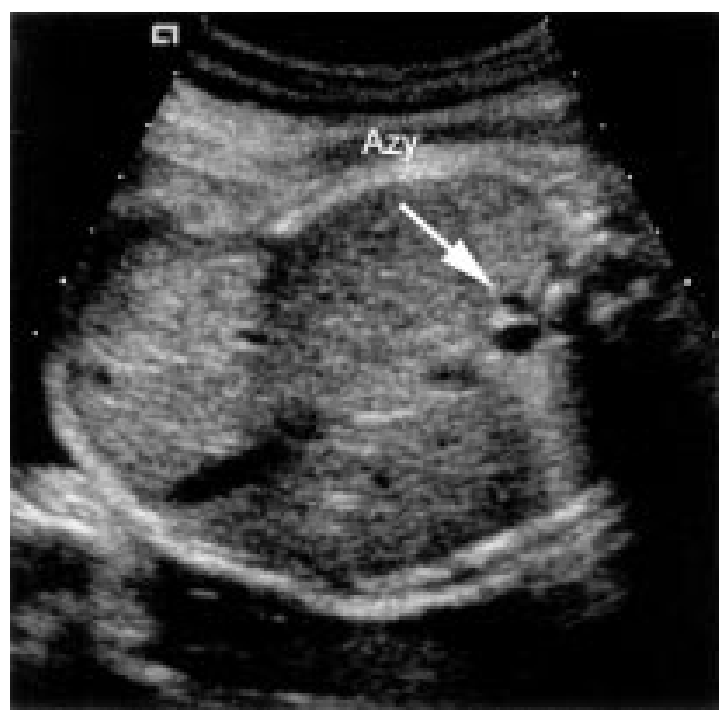

Figura 3. Situs vascular abdominal en un caso de interrupción de $\mathrm{VCl}$ e interrupción azygos. La flecha indica la vena a derecha y posterior de la aorta abdominal. condiciones normales y de izquierda a derecha: el tronco de la arteria pulmonar, la aorta ascendente y la vena cava superior (VCS). Los dos últimos vasos se observan en sección transversa, la vena cava superior de menor tamaño que la aorta ascendente. Cuando la VCl está interrumpida y el retorno es por el sistema Azygos, esta vena desemboca a nivel de la VCS. El tamaño de este vaso será mayor que en condiciones habituales (Figura 5). Un sutil movimiento del transductor a este nivel permitirá confirmar la desembocadura de la $\mathrm{Az}$ hacia la VCS.

Durante el estudio postnatal, la ecografía abdominal confirmó ausencia de la vena cava inferior sobre las venas renales, visualizándose en 3 de los 4 casos la vena azygos dilatada. No se visualizaron anomalías esplénicas en ninguno de los 4 casos. Todos los recién nacidos fueron evaluados por cardiólogo infantil, efectuándose un estudio

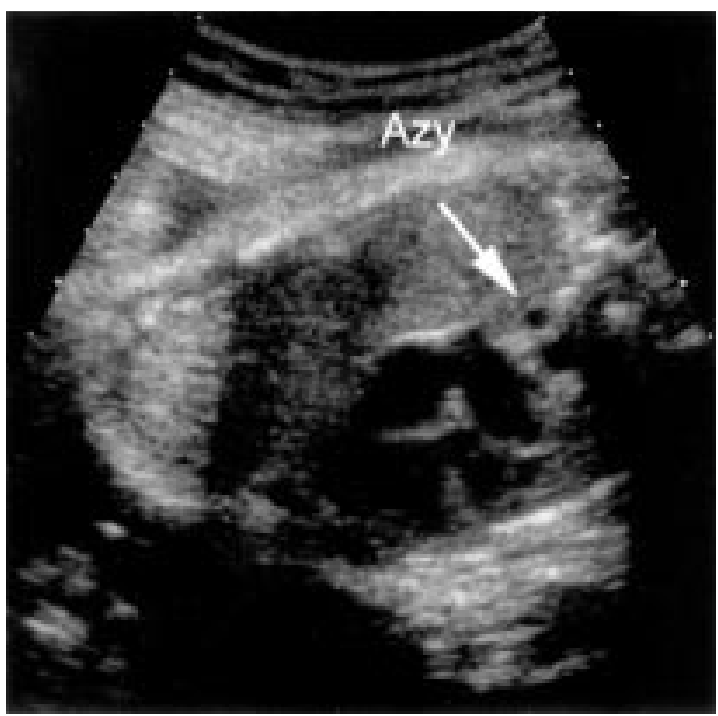

Figura 4. A nivel de 4 cámaras, se visualiza un "segundo vaso" por detrás de la aurícula izquierda fetal. En condiciones fisiológicas sólo se observa la aorta torácica. 


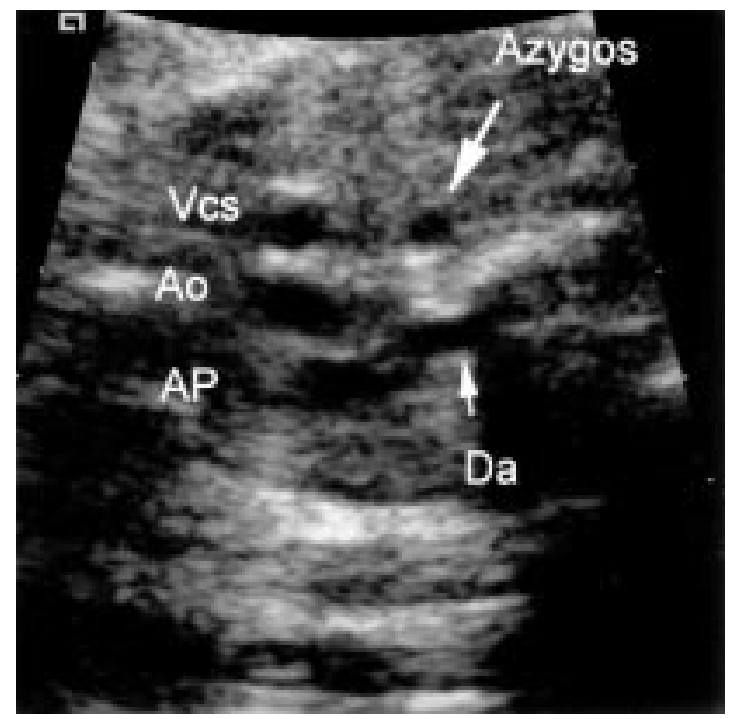

Figura 5. En la sección de "3 vasos", se visualiza la vena cava superior (Vcs) de mayor tamaño, debido al drenaje de la azygos. La flecha indica la vena azygos como un vaso extra a este nivel (Ao: aorta; AP: tronco de la arteria pulmonar; DA: ductus arterioso).

ecocardiográfico en 3 de los 4 casos. En 2 de estos se confirmó que la cava inferior no drenaba a la aurícula derecha, descartándose defectos cardíacos asociados.

Todos los recién nacidos están vivos al día de hoy, sin anomalías pesquisadas en controles posteriores. La única anomalía extracardíaca diagnosticada in utero y confirmada postnatal fue en el caso 1 y se trataba de un riñón único. El caso 4 fue derivado a ecografía por hipomotilidad fetal asociada a una bradicardia relativa de 110-115 por minuto, mantenida durante días. Además de los hallazgos de la interrupción $\mathrm{VCl}$, existía un discreto engrosamiento miocárdico y un mínimo derrame pericárdico. No se visualizaron defectos estructurales cardíacos in utero y la evolución hasta 4 meses postparto ha sido satisfactoria.

Incluimos un caso de isomerismo auricular derecho diagnosticado el año 2002, en una paciente de 31 años que consultó por primera vez a nuestro centro a las 31 semanas de gestación sin sospecha previa. Diagnosticamos un canal atrioventricular desbalanceado, transposición de las grandes arterias, drenajes anómalo pulmonar total y estenosis pulmonar. El situs vascular abdominal reveló una $\mathrm{VCl}$ por delante de la aorta abdominal (Figura 7). Esto confirma la importancia de visualizar estas estructuras vasculares y su relación en cada exploración ecográfica.

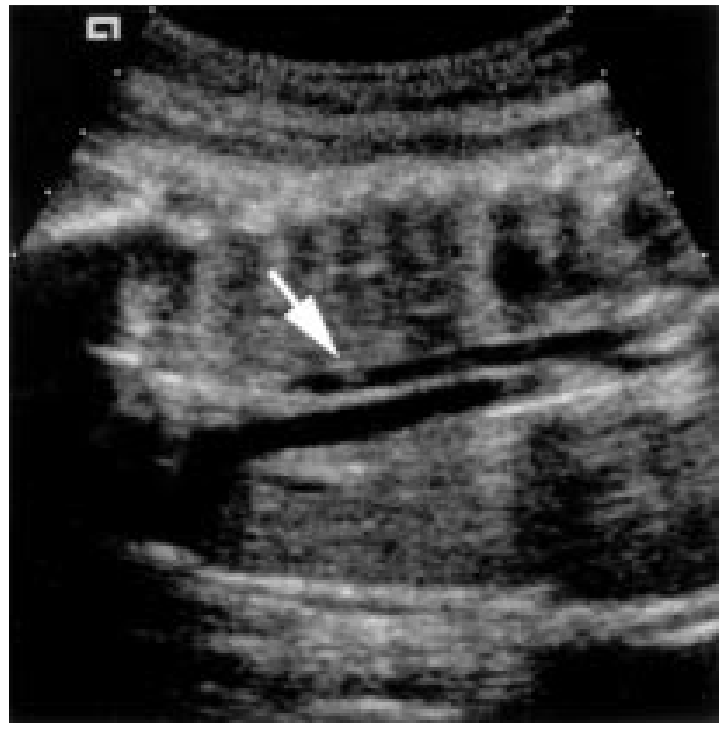

Figura 6. En un corte sagital del abdomen de un feto con interrupción de la $\mathrm{VCl}$ y continuación azygos, se visualizan "2 vasos" paralelos. La flecha indica el curso de la vena azygos.

\section{DISCUSION}

La visualización de la $\mathrm{VCI}$ en la sección transversa del abdomen fetal es la forma más simple y efectiva de sospechar anomalías de esta porción del sistema venoso (23). No sólo importa si está presente o ausente, sino que además su posición

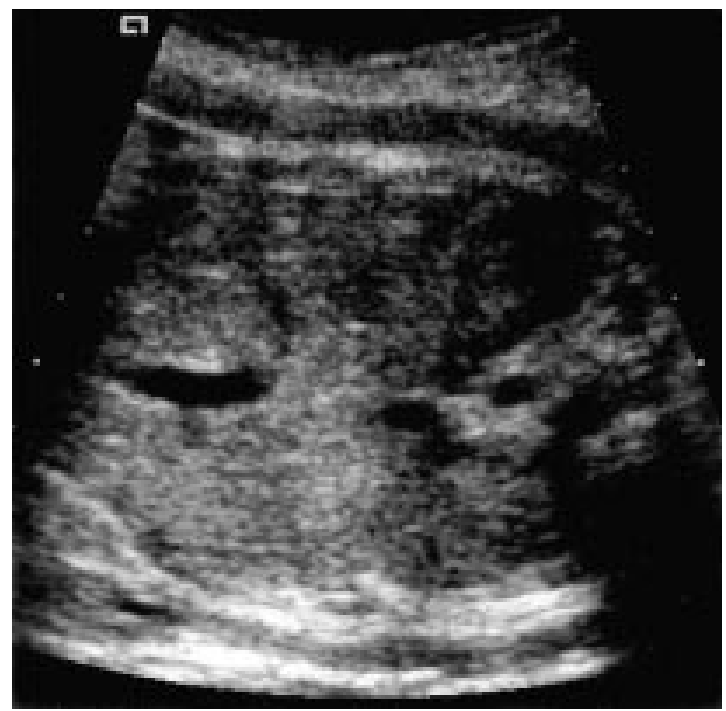

Figura 7. Situs vascular abdominal en un caso de isomerismo auricular derecho. La VCI se localiza por delante de la aorta y más medial que lo habitual. 
Tabla III

\section{INCIDENCIA DE ANOMALIAS CARDIOVASCULARES EN SINDROMES DE ASPLENIA Y POLIESPLENIA}

\begin{tabular}{lcc}
\hline & Asplenia & Poliesplenia \\
\hline Defecto interauricular & $90 \%$ & $80 \%$ \\
Canal atrioventricular & $85 \%$ & $40 \%$ \\
Ausencia de VCl & Raro & $80 \%$ \\
Vena cava superior bilateral & $50 \%$ & $40 \%$ \\
Dextrocardia & $40 \%$ & $40 \%$ \\
Doble tracto salida derecho & $80 \%$ & $40 \%$ \\
Drenaje anómalo pulmonar parcial & Raro & $40 \%$ \\
Drenaje anómalo pulmonar total & $70 \%$ & Raro \\
Estenosis/atresia pulmonar & $80 \%$ & $30 \%$ \\
Ventrículo único & $50 \%$ & $10 \%$ \\
Transposición grandes arterias & $80 \%$ & $30 \%$ \\
\hline
\end{tabular}

*Adaptado de: Hobbins JC, Drose JA: Cardiosplenic syndromes. En Drose: Fetal echocardiography. $1^{\text {st }}$ ed. WB Saunders \& Co 1998; 254.

en relación con la columna vertebral fetal y la aorta abdominal. Consideramos que el situs vascular a este nivel es un marcador que orienta a la sospecha diagnóstica de anomalías cardíacas, especialmente las relacionadas con síndromes de isomerismo. Si en condiciones normales el situs solitus corresponde al ordenamiento normal de las aurículas cardíacas y las vísceras, el situs inversus corresponde a la situación "en espejo". La tercera posibilidad corresponde a cualquier otro tipo de ordenamiento, denominándose esto situs ambiguo $(7,24)$. Los síndromes que presentan anormalidades de lateralización del tipo ambiguo han recibido los nombres de heterotaxia, asplenia, poliesplenia, síndrome de Ivemark, así como también isomerismo auricular. En general, el síndrome de asplenia se relaciona con el isomerismo de la aurícula derecha y el de poliesplenia con el isomerismo izquierdo (14). El término de isomerismo es el más apropiado y habitualmente utilizado para referirse a estos cuadros.

Tal como se enumeran en la Tabla III, se asocian comúnmente a anormalidades estructurales del corazón fetal (15). La incidencia es dificil de calcular, pero grandes series indican que cerca del $4 \%$ de los casos de Cc corresponde a isomerismos (16). Aunque su incidencia es baja, se caracterizan por ser diagnósticos difíciles, en corazones portadores de más de un defecto. Más aún para el operador no experimentado que se alarma por la presencia de una anomalía mayor de las 4 cámaras o una alteración significativa del ritmo cardíaco. Sin embargo, la observación de la $\mathrm{VCl}$ ayuda a resolver muchas de estas dificultades. En la Fi-

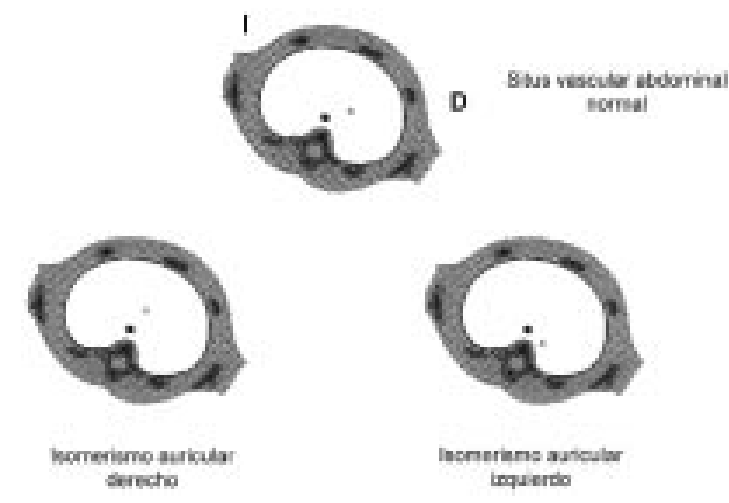

Figura 8. Situs vascular (abdomen transverso) en condiciones fisiológicas y en anormalidades de lateralización (círculo negro: aorta abdominal; círculo blanco: vena cava inferior).

gura 8 se describen las alteraciones posibles de visualizar en la sección de abdomen transverso, en condiciones normales y en casos de isomerismo auricular derecho e izquierdo. La interrupción de la $\mathrm{VCl}$ y continuación azygos está presente en casi el $80 \%$ de los casos de isomerismo izquierdo; en el derecho, es habitual visualizar la VCI por delante de la aorta y no a derecha de la línea media.

Las lesiones cardíacas asociadas comúnmente a la interrupción de la $\mathrm{VCl}$ y continuación azygos son: canal atrioventricular, anomalías de conexión de las venas pulmonares, doble tracto de salida derecho, comunicación interauricular amplia, estenosis o atresia pulmonar, posición anormal del corazón fetal y anomalías de la vena cava superior (5). Todas ellas se pueden incluir en el isomerismo auricular izquierdo. Los hallazgos ecográficos más frecuentes de este cuadro se enumeran en la Tabla IV.

Tabla IV

\section{CARACTERISTICAS ECOGRAFICAS FRECUENTES} DEL ISOMERISMO AURICULAR IZQUIERDO

- Eje cardíaco hacia izquierda o más central

- Vena cava inferior no visible en porción hepática (80\%)

- Venas hepáticas drenan directamente hacia la aurícula

- Dos vasos detrás del corazón

- Habitualmente, canal atrioventricular

- Puede haber bloqueo A-V completo (68\%)

- Corazón dilatado e hipertrófico (con bloqueo)

- Vena cava superior izquierda persistente (56\%)

- Drenaje anómalo venoso pulmonar 
El método clásico para la confirmación diagnóstica es la angiografía venosa desde las venas de las extremidades inferiores $(3,10)$. Estudios recientes demuestran la utilidad de técnicas no invasivas como la tomografía axial computada (17). Los síntomas clínicos son dependientes de la presencia de anomalías cardíacas asociadas. En caso que el defecto sea aislado, no hay síntomas.

El consejo antenatal y manejo perinatal de los casos de interrupción de la $\mathrm{VCl}$ dependerá fundamentalmente de la presencia de anomalías cardíacas asociadas. En los casos aislados, el tamaño de la vena es adecuado para el apropiado retorno venoso, por lo que la fisiología es normal, sin existir consecuencias clínicas durante la vida postnatal. Sin embargo, su conocimiento es importante para la preparación preoperatoria de bypass cardiopulmonares o para evitar dificultades durante cateterismos cardíacos futuros $(18,19)$.

A pesar que no se adviertan defectos cardíacos durante la vida intrauterina, el estudio postnatal debe siempre incluir una ecocardiografía y una evaluación ecográfica abdominal, buscando dirigidamente malformaciones no pesquisadas a nivel cardíaco y las alteraciones extracardíacas asociadas a la poliesplenia $(20,21)$. La asociación de interrupción de la $\mathrm{VCI}$ con canal auriculoventricular, bloqueo aurículoventriculr completo y poliesplenia se asocia a elevada mortalidad perinatal.

Como conclusión, creemos que la visualización del situs vascular de la sección transversa del abdomen fetal es una importante herramienta a incluir en todas las evaluaciones ecográficas fetales. Considerando que los reparos anatómicos en condiciones fisiológicas son simples, las anormalidades pesquisadas permiten seleccionar a un grupo de fetos con riesgo de presentar Cc severas. Mediante el estudio ecocardiográfico posterior y el apropiado consejo perinatal a la pareja, se logrará disminuir el impacto que la Cc tendrá en ese recién nacido.

AGRADECIMIENTOS: Nuestros agradecimientos al Dr. Rodrigo Nazal, Cardiólogo Infantil, por su colaboración en la evaluación ecocardiográfica postnatal.

\section{BIBLIOGRAFIA}

1. Ghi T, Huggon IC, Zosmer N, Nicolaides KH: Incidence of major structural cardiac defects associated with increased nuchal translucency but normal karyotype. Ultrasound Obstet Gynecol 2001; 18: 610-4.
2. Zosmer N, Souter VL, Chan CSY, Huggon IC, Nicolaides K: Early diagnosis of major cardiac defect in chromosomally normal fetus with increased nuchal translycency. Br J Obstet Gynaecol 1999; 106: 82933.

3. Adams FH, Emmanouiliades GC: Moss heart disease in infants, children and adolescents. $5^{\text {th }}$ ed. Baltimore: Williams and Wilkins 1995; 890-4.

4. Stevenson RE, Hall JG, Goodman RM: Human malformations and related anomalies. $1^{\text {st }}$ ed. Oxford: University Press, Inc 1993; 265.

5. Anderson RC, Adams P, Burke B: Anomalous inferior vena cava with azygous continuation (infrahepatic interruption of the inferior vena cava). J Pediatr 1961; 59: 370 .

6. Ramsaren EK, Filiberti AW, Spodick DH: Isolated anomalous inferior vena cava with azygos continuation. Cardiology 1995; 86: 257.

7. Sharland G, Cook A: Heterotaxy syndromes/isomerism of the atrial appendages. In: Allan LD, Homberger L, Sharland GK (eds). Fetal Cardiology. London: Greenwich Med Publish 2000; 333-46.

8. Anderson C, Devine WA, Anderson RH, Debich DE, Zuberbuhler JR: Abnormalities of the spleen in relation to congenital malformations of the heart: a survey of necropsy findings in children. Br Heart $\mathrm{J}$ 1990; 63: 122-28.

9. J Phoon CK, Villegas MD, Ursell PC, Silverman NH: Left atrial isomerism detected in fetal life. Am J Cardiol 1996; 77: 1083-8.

10. Stackelberg B, Lind J, Wegelius C: Abscence of inferior vena cava diagnosed by angiocardiography. Cardiology 1952; 21: 583.

11. Viñals F, Tapia J, Giuliano A: Exploración del corazón fetal. ¿Cómo simplificar las cosas? REV CHIL OBSTET GINECOL 1999; 4: 275.

12. Viñals F, Tapia J, Giuliano A: Diagnóstico antenatal de defectos ventriculoseptales. A propósito de nuestra técnica, experiencia y consideraciones perinatales. Rev Chil OBstet GineCOL 2000; 3: 177-82.

13. Viñals F, Tapia J, Giuliano A: Prenatal detection of ductal-dependent congenital heart disease: how things can be made easier? Ultrasound Obstet Gynecol 2002; 19: 246-9.

14. Macartney FJ, Zuberbuhler JR, Anderson RH: Morphological considerations pertaining to recognition of atrial isomerism. Consequences of sequential chamber localization. Br Heart J 1980; 44: 657-67.

15. Hobbins JC, Drose JA: Cardiosplenic syndromes. In: Drose JA: Fetal Echocardiography. $1^{\text {st }}$ ed. Philadelphia: WB Saunders \& Co 1998; 253-62.

16. Fyler DC, Buckley LP, Hellenbrand WE, Cohn HE: Report of the New England Regional Infant Care Programe. Pediatrics 1980; 65(Suppl): 376-61.

17. Bass JE, Redwine MD, Kramer LA, Huynh PT, Harris $\mathrm{JH}$ : Spectrum of congenital anomalies of the inferior vena cava: cross-sectional imaging findings. Radiographics 2000; 20: 639-52.

18. Effler DB, Greer AE, Sifers EC: Anomaly of the vena 
cava inferior: report of fatality after ligation. J Am Med Assoc 1951; 146: 1321

19. Parikh SR, Prasad K, Lyer RN, Desai N, Mohankrishna L: Prospective angiographic study of the abnormalities of systemic venous connections in congenital and acquired hearth disease. Cathet Cardiovasc Diagn 1996; 38: 379-86.

20. Caruso G, Becher AE: How to determine atrial situs? Considerations initiated by 3 cases of absent spleen with discordant anatomy between bronchi and atria. Br Heart J 1979; 41: 559-76.

21. Uemura $\mathrm{H}$, Ho SY, Devine WA, Anderson $\mathrm{RH}$ : Analysis of visceral heterotaxy according to splenic status, appendage morphology or both. Am J Cardiol 1995; 76: 846-9.

22. K Sheley RC, Nyberg DA, Kapur R: Azygous continuation of the interrupted inferior vena cava: a clue to prenatal diagnosis of the cardiovascular syndromes. J Ultrasound Med 1995; 14: 381-7.

23. Huhta JC, Smallhom JF, Macartney FJ: Two dimensional echocardiographic diagnosis of situs. $\mathrm{Br}$ Heart J 1982; 48: 97-108.

24. Hofstaetter $\mathrm{C}$, Plath $\mathrm{H}$, Hansmann M: Prenatal diagnosis of abnormalities of the fetal venous system. Ultrasound Obstet Gynecol 2000; 15: 231-41. 